\title{
Balance Assessment during the Landing Phase of Jump-Down in Healthy Men and Male Patients after Anterior Cruciate Ligament Reconstruction
}

\author{
Aleksandra Melińska', Andrzej Czamara', Lukasz Szuba², \\ Romuald Będziński ${ }^{3}$, Ryszard Klempous ${ }^{4}$ \\ ${ }^{1}$ Wrocław University of Technology, Faculty of Fundamental Problems of \\ Technology, Wybrzeże Wyspiańskiego 27, 50-370 Wrocław, Poland \\ aleksandra.melinska@pwr.edu.pl \\ ${ }^{2}$ College of Physiotherapy in Wroclaw, T. Kościuszki 4, 50-038 Wrocław, Poland \\ a.czamara@wsf.wroc.pl \\ ${ }^{2}$ College of Physiotherapy in Wroclaw, T. Kościuszki 4, 50-038 Wrocław, Poland \\ lukasz.szuba@wsf.wroc.pl \\ ${ }^{3}$ University of Zielona Góra, Faculty of Mechanical Engineering, Biomedical \\ Engineering Group, ul. Licealna 9, 65-417, Zielona Góra, Poland \\ romuald.bedzinski@pwr.edu.pl \\ ${ }^{4}$ Wrocław University of Technology, Faculty of Electronics, Wybrzeże \\ Wyspiańskiego 27, 50-370 Wrocław, Poland, ryszard.klempous@pwr.edu.pl
}

\begin{abstract}
The aim of the study was to evaluate balance during the landing phase in a control group and patients after anterior cruciate ligament reconstruction (ACLR). ACL tear is a frequent injury, particularly in athletes. It often requires proper rehabilitation, during which time the patients should be re-evaluated. We performed a jump-down assessment using a motion capture and force platform system. The tests comprised a series of time-space and dynamic parameters measurements using a motion capture system and a force plate. We tested 28 men (22 controls and 6 patients who had undergone ACLR). The tests are conducted under jump-down conditions (0.1-, 0.2-, and 0.3-m step heights). We compared horizontal components of force during the landing phase. The division of registered three-dimensional (3D) motion was demonstrated, and the fluctuations of the estimated center of gravity (eCOG) during this motion were analyzed. Compared with the controls, patients showed statistically significant differences in fluctuations in the eCOG repositioning and horizontal components of force ratios (t-test, $p<0.05)$. We propose a comparative assessment of balance attainment during the landing phase of a jump. We indicate the possibility of efficient detection of ACL injuries. In summary, horizontal ground reaction forces and eCOG positioning can be used to evaluate the performance of the human biomechanical system during the jump-down phase.
\end{abstract}


Keywords: Ground reaction forces; Body mass center repositioning; ACLR; Motion analysis

\section{Introduction}

Injuries of the anterior cruciate ligament (ACL) are common in athletes, but they also occur in other individuals, where permanent uneven loading of lower limbs is observed [1-4]. Mechanical failure of the ACL necessitates ligament reconstruction surgery (ACLR), often leading to lengthy rehabilitation that ensures stabilization of the knee joint and proper force distribution. Monitoring rehabilitation progress is obligatory for assessing its effectiveness. Motion analysis measurements and techniques are helpful in this regard.

There have been numerous motion analysis studies, but they are mainly concerned with gait [5-13]. Some studies considered motion analysis after ACLR [14-16]. In cases of lower limb dysfunction, such as those requiring ACLR, motion assessment is possible not only for gait conditions but also during running or jump-down [17-31]. Jump-down is a natural activity for humans (e.g., getting down a staircase), and the amount of loading differs between walking and running. Jump-down has another interesting aspect as well: the landing phase. Among the studies that considered jump-down, some of the important ones addressed only the landing strategy. The landing phase is where the subject attains balance, so the landing mechanisms should be carefully observed [32-36]. The motion capture system, force plates, and electromyography (EMG) are useful for assessing balance. It is possible to assess balance by measuring the ground reaction force, segments of the body's repositioning, the angles between segments, and muscle activity [37-40].

We suggest that there is a need to broaden studies concerning the identification of load that occurs during the landing phase of jump-down. The research scope includes analysis of 3D data derived from dynamometric platforms and the motion capture system. Unlike the existing studies, we focused here on the horizontal components of the ground reaction force and the estimated body's mass center of gravity (eCOG) repositioning during the landing phase. The aim of the study was to evaluate balance during the landing phase in a control group and patients after anterior cruciate ligament reconstruction (ACLR). Our hypothesis was: Is the balance characteristics during the landing phase different in the normal controls and patients who underwent ACLR? 


\section{Methods}

A total of 28 men aged 20-26 years were studied. In all, 22 of them did not show any abnormality and were not diagnosed with any knee joint dysfunction. They constituted the control group. The other six men, who had undergone ACLR of the left limb, comprised the patient group. These six patients had gone through a 3month supervised rehabilitation course and then carried out a rehabilitation program on their own. The ACLR rehabilitation program was performed according to the protocol developed by Czamara et al. [41]. The patients agreed to participate in the study during the 8th month after their surgery. The control group was matched for age, body height, and body mass. Data characterizing the groups (mean \pm SD) were as follows: controls: age $25.1 \pm 4.3$ years, height $1.78 \pm 0.19 \mathrm{~m}$, weight $78.5 \pm 9.7 \mathrm{~kg}$; patients: age $26.2 \pm 2.3$ years, height $1.72 \pm 0.91 \mathrm{~m}$, weight $74.3 \pm 5.6 \mathrm{~kg}$. All subjects were treated according to the tenets of the Declaration of Helsinki.

Before the examination, each person was informed about the course of the study and was requested to consent to participation. The tests comprise a series of time-space and dynamic parameters measurements. The individual tests consisted of a jump-down from three step heights $(0.1,0.2$, and $0.3 \mathrm{~m})$. After jump-down from $0.1 \mathrm{~m}$, another $0.1 \mathrm{~m}$ was added to the platform until the height of $0.3 \mathrm{~m}$ was obtained. There was a 30 -second break between the jumps. The baseline position for each participant was free standing on both legs on the step. On the examiner's command, the participant jumped down. Each subject was instructed that he should land on his toes and metatarsus on both limbs (one limb per platform). The tests were preceded by a short warm-up involving trotting (3-4 minutes), five to seven jumps, and (after a 20-s interval) four to five squats. After a short break, each subject performed trial jumps from different heights. The jumps were followed by a 2-min rest break.

A dynamometric platform and a motion capture system were used for the tests. Body center repositioning was registered using a module of the BTS Smart (BTS Bioengineering, Milan, Italy). BTS Smart-D3 is a module that performs the functions of multi-channel 3D navigation. It operates on the basis of an image composition from six infrared cameras that capture movement of markers placed on the body. For this study, we used one marker placed on the skin near the sacral bone to estimate the location of the COG. The ground reaction forces during a jump were measured by two dynamometric platforms (one for each leg) onto which the subject jumped. The output signal from each platform included the resultant force vector, component vectors, magnitude, and force direction. The measuring system is shown in Figure 1. The test also involved measuring ground reaction forces and the eCOG repositioning while free standing before each jump. 


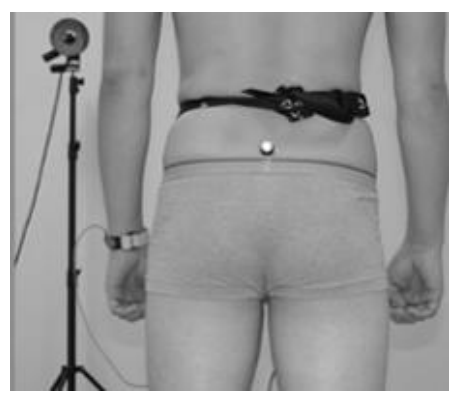

Figure 1

Data acquisition setup

The force during jump-down was measured from the first contact of the feet with the platform. Ground reaction force was measured in three directions. The motion was divided into two phases. Examples of force outputs divided into phases are shown in Figure 2 for one control group and one patient.
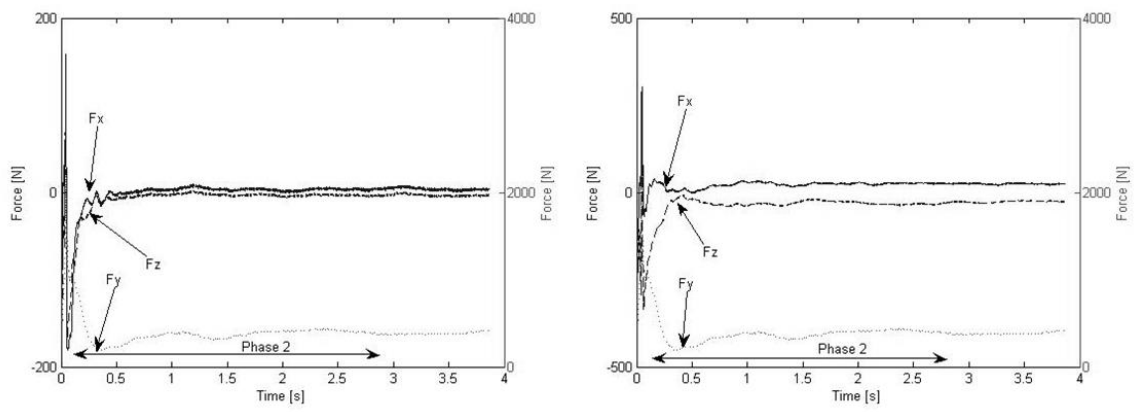

Figure 2

Force signals for one healthy control (left) and one patient (right)

The first phase covered the period from the initial contact of the foot with the surface (the time at which the vertical value of the ground reaction force, Fy, is minimum) to the time when Fy is maximum. This phase was not considered in our analysis because the meaningful phase of the jump-down is the landing phase (phase 2). The landing phase begins at the time the vertical components of force reach their maximum. It covers the period when the values of force do not vary by more than $20 \%$ of the maximum value. This occurs approximately $3 \mathrm{~s}$ after the jump on the platform. Note that the vertical force is not a part of this study. (We earlier reported a separate study on vertical ground reaction forces [42]). Here, the horizontal vectors were examined because they are responsible for balance. They are calculated as follows:

$$
\pi_{F}=\frac{\overline{F_{x}}}{\overline{F_{z}}}
$$


where $\bar{F}=\frac{1}{n} \sum_{p}^{n-p} F_{s}^{*} F$ is the average force component, subscripts $x$ and $z$ correspond to the horizontal components of force, $p$ is the number of observations, $F_{y}$ is the maximum force, and $n$ is the number of samples.

Force is not the only value that is important during the landing phase. Body repositioning is crucial as well. The eCOG position can be used to describe balance during landing. The traction is registered by cameras in the motion capture system. The eCOG position is registered in 3D space in the time domain. The 3D motion is divided into two orthogonal planes: horizontal and vertical. This concept is shown in Figure 3.
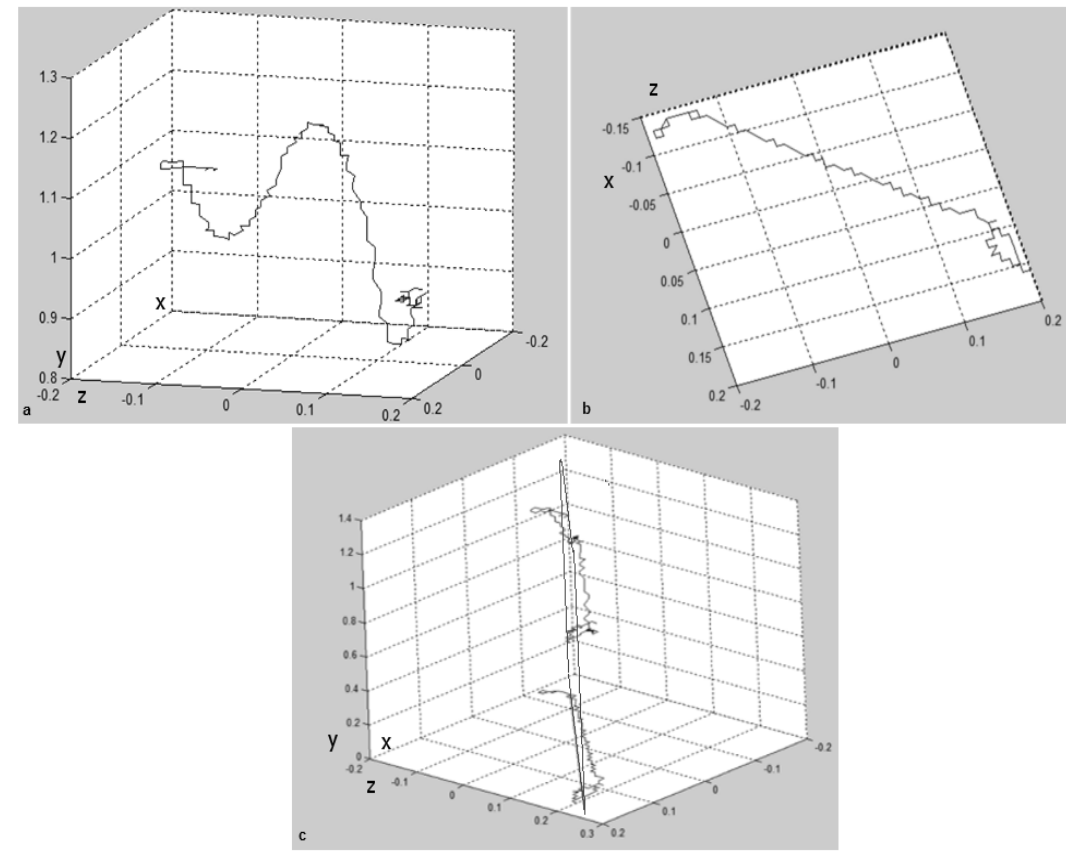

Figure 3

Concept of motion: a) 3D motion traction (XYZ), b) Projection for plane XZ c Projection for the XZ plane with a regressive trajectory

We divided the trajectory function into $f\left(x_{v}, y_{v}, t\right)$ for (1) projection horizontal components $p(x, z)(t)$ and (2) representation of the vertical component in the common time domain $y(t)$.

The registered trajectory in 3D space is a function of time $t$ and the marker position $x, y, z$ in axes: 
$f(x, y, z, t)=\left\{\begin{array}{l}p(x, z) \\ r(p, x)\end{array}\right.$

where ${ }^{p}$ finds coefficients of a first-degree polynomial that best fits the input data $(p(x(t), z(t))$ in an orthogonal least-squares sense. The projected trend is given by $p=m x+n(2)$, which returns the predicted value of the polynomial, given its coefficients, to values in $x$.

Next, we found the distance $d$ from a point $\left(x_{0,} y_{0}\right)$ to a line $(a x+b y+c=p=m x+n)$ for each value in the time domain.

The parametric equation of a straight line is given as:

$$
d=\frac{|m x+n y+c|}{\sqrt{m^{2}+n^{2}}}
$$

where $m, n, c$ are real constants satisfying $\mathrm{m}, \mathrm{n} \neq 0$

Results are presented on the two-axes chart $f=$ (time, $d_{;}$time,y).

The eCOG fluctuations in the controls and patients were analyzed. Two approaches for measuring those fluctuations are proposed:

a) Determining the average eCOG fluctuation,

$$
\bar{d}_{t}=\frac{\sum_{\mathrm{t}=0}^{n} d_{\mathrm{t}}}{n}
$$

where $d_{t}$ is the distance between the eCOG to the regressed projected trajectory registered in time $t$, where $n$ is the number of samples.

b) Determining the sum of the eCOG fluctuation from the regressive trajectory.

$$
d_{t}=\sum_{t=0}^{n} d_{t}
$$

where $d_{t}$ is the distance between the eCOG to the regressed projected trajectory registered in time $t$, where $n$ is the number of samples.

Statistical analysis was conducted using SPSS (IBM SPSS Statistics for Windows, Version 22.0. Armonk, NY: IBM Corp, Released 2013). We conducted t-test for assessing if there are significant differences for patients and control group in horizontal components force ratio in landing phase and also in free standing (Table 1). We used two-way ANOVA with Bonferroni correction to test if there are differences in fluctuation of eCOG in patients and control group as well as if there is interaction between step high and measured parameters (Table 2). 


\section{Results}

Table 1 shows the horizontal components force ratios during the landing phase of the jump and free-standing values (mean $\pm \mathrm{SD}$ ). In case of the control group, the model horizontal components force ratio during jump-down should be similar to those observed in the free-standing condition [37]. The average force ratio increases with the step height increase. The force ratios in free-standing phase for the control group and patients are not significantly different, as well as there is no statistically significant difference between right and left leg for the control group, and there are for patients (t-test, $\mathrm{p}>0.05$ ). There are statistically significant differences between patients and the control group for right leg and for the left leg in case of 0.2 and $0.3 \mathrm{~m}$ step height (t-test, $\mathrm{p}<0.05$ ).

Table 1

Horizontal components ratio of ground reaction forces

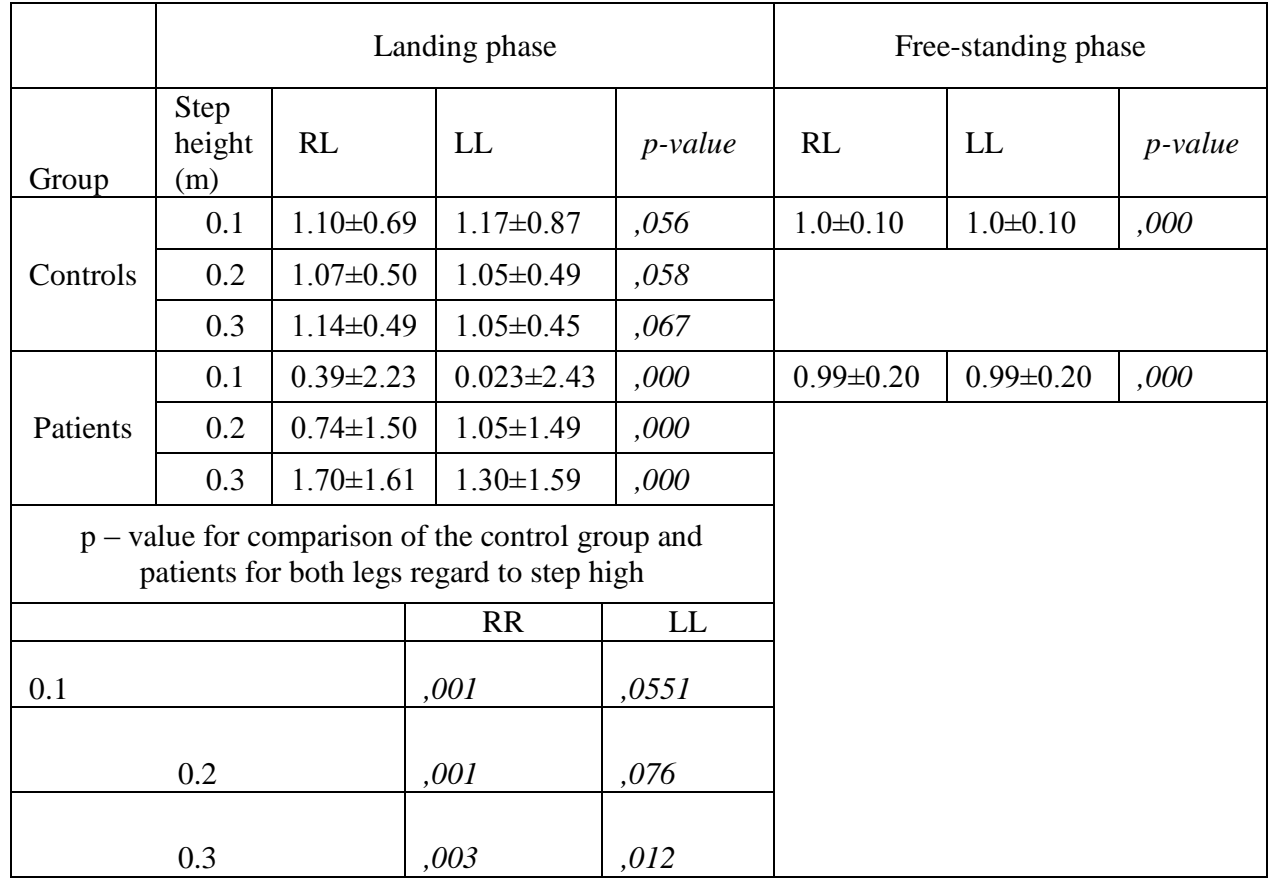

$\mathrm{LL}=$ left limb, $\mathrm{RL}=$ right limb

Fluctuations from eCOG trend motion were analyzed in the same way for both the control group and those who had undergone ACLR. An example of the results of differences between the control group and patients is shown in Figure 4, represented by the measurements for one healthy control and one ACLR patient for the 0.1-m step. 

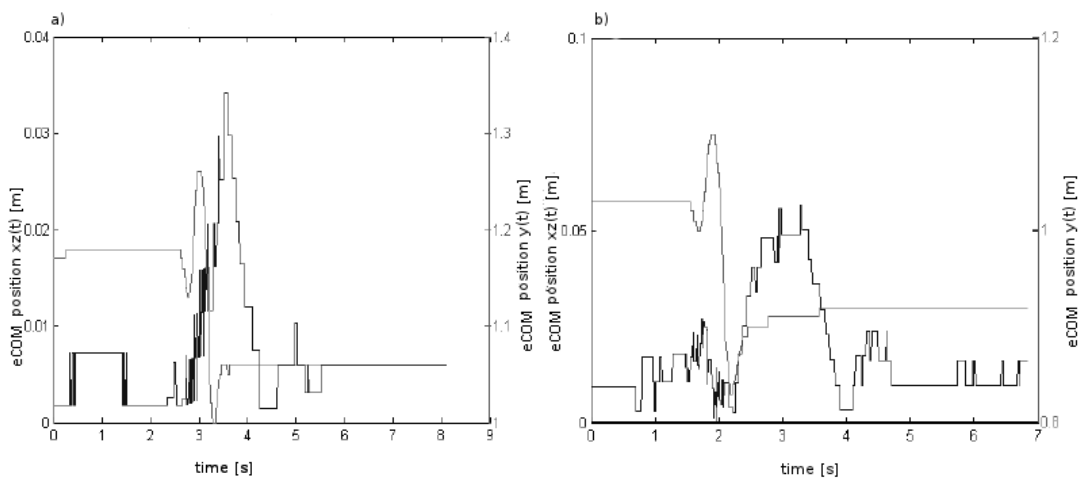

Figure 4

Example of eCOG fluctuation for a healthy control and an ACLR patient. The jump was from $0.1 \mathrm{~m}$.

We propose two measures for evaluating balance: the sum and an average of the fluctuation of the eCOG from the regressed motion trajectory during the patients' jumps (equations 1, 2, 3). The results are shown in Figures 5 and 6.

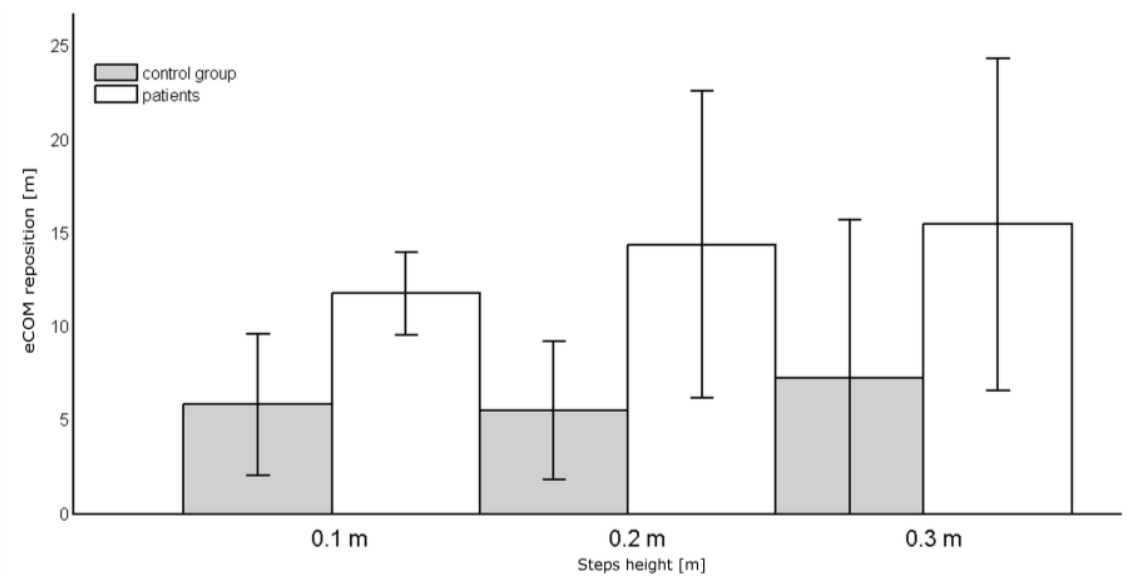

Figure 5

Sum of fluctuation of distance of the eCOG from a regressed motion trajectory in the horizontal plane

Both results show that the measured fluctuations are substantially greater for the patients than for the controls. The step height had an effect on the patients' results. The sum increased with greater step height for patients although the arithmetic mean decreases. The step height had no effect on the healthy controls. Healthy people land more stably than patients, which we proved in the final statistics. The sum of the fluctuation increased with step height for the ACLR patients. Using ANOVA test we checked if there is interaction between step height and fluctuations of eCOG (average and sum of fluctuation). There are statistical significant differences for patients for the step height 0.2 and $0.3 \mathrm{~m}$. 


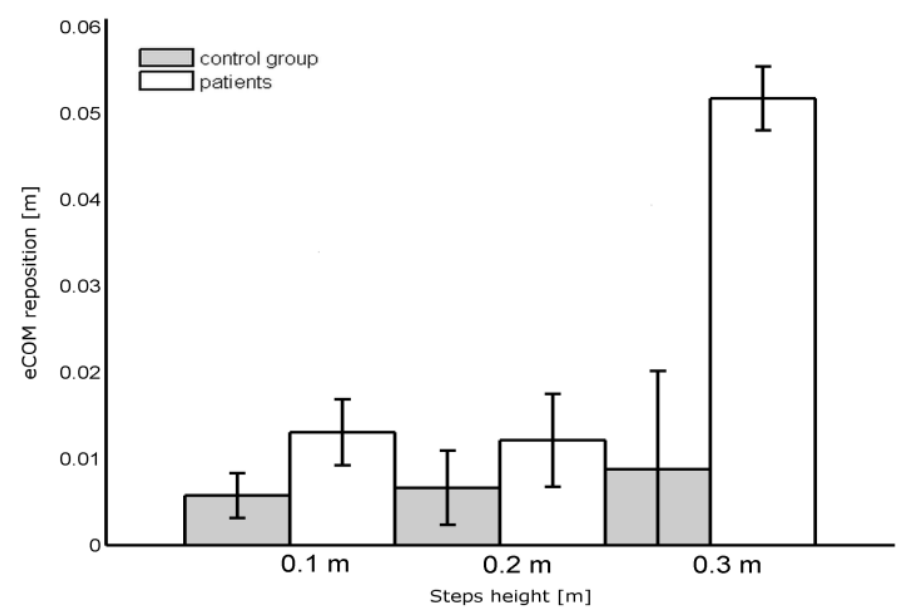

Figure 6

Average sum of the distance of the eCOG from a regressed motion trajectory in the horizontal plane

Table 2

Statistics for ANOVA test for eCOG fluctuation measures

\begin{tabular}{|c|c|c|c|}
\hline \multirow{7}{*}{$\begin{array}{l}\text { Sum of } \\
\text { fluctuations } \\
\text { of distance of } \\
\text { the eCOG }\end{array}$} & (I) step high & (J) step high & p-value \\
\hline & \multirow[t]{2}{*}{0.1} & 0.2 & \multirow[t]{2}{*}{, 054} \\
\hline & & 0.3 & \\
\hline & \multirow[t]{2}{*}{0.2} & 0.1 & \multirow[t]{2}{*}{,017 } \\
\hline & & 0.3 & \\
\hline & \multirow[t]{2}{*}{0.3} & 0.1 & \multirow[t]{2}{*}{,017 } \\
\hline & & 0.2 & \\
\hline \multirow{7}{*}{$\begin{array}{l}\text { Average of } \\
\text { the } \\
\text { fluctuation of } \\
\text { the eCOG }\end{array}$} & (I) step high & (J) step high & p- value \\
\hline & \multirow[t]{2}{*}{0.1} & 0.2 & \multirow[t]{2}{*}{,066 } \\
\hline & & 0.3 & \\
\hline & \multirow[t]{2}{*}{0.2} & 0.1 & \multirow[t]{2}{*}{,012 } \\
\hline & & 0.3 & \\
\hline & \multirow[t]{2}{*}{0.3} & 0.1 & \multirow[t]{2}{*}{,012 } \\
\hline & & 0.2 & \\
\hline
\end{tabular}




\section{Discussion}

This study evaluated the jump-down phase by analyzing horizontal components of the ground reaction force and eCOG repositioning during the landing phase. Several other studies have considered jump-down [23, 24, 38, 42-47]. Among them, some are important from the point of view of balance.

Paterno et al. [43] presented a case-control study of female athletes at a mean of 27 months following their ACLR versus healthy female subjects. All of the participants executed a task that comprised a drop vertical jump onto two force plates (one per leg). The vertical ground reaction force, which was measured during landing and takeoff, was used to calculate the landing phase loading rates. The authors concluded that female athletes who have undergone ACLR and return to their sport may continue to demonstrate biomechanical limb asymmetry 2 years or more after reconstruction. It can be identified during landing.

Similarly, using force plates, Ortiz et al. [44] demonstrated the landing mechanics in noninjured women and women with ACLR. They performed five trials of a single-leg 40-cm drop jump and two trials of a 20-cm up-down hop task. Multivariate analyses of variance were used to compare hip and knee joint kinematics, knee joint moments, ground-reaction forces, and EMG findings between the dominant leg in noninjured women and the reconstructed leg in women who had undergone ACLR. The results of their study showed that women with ACLR have neuromuscular strategies that allow them to land from a jump similar to healthy women, but they exhibit joint moments that could predispose them to future injury if they participate in sports that require jumping and landing.

Decker et al. [45] purposed their study to determine whether fully rehabilitated ACLR recreational athletes utilize adopted lower-extremity joint kinematics and kinetics during high-demand functional tasks. Subjects were compared during a $60-\mathrm{cm}$ vertical drop landing. The hamstring ACLR recreational athletes used an adapted landing strategy that employed less of the hip extensor muscles and more of the ankle plantar flexor muscles. Harvesting the medial hamstring muscles for ACL reconstruction may contribute to the utilization of this protective landing strategy.

Gribble and Hertel et al. [46] designed their study to examine the role of foot type, height, leg length, and range of motion (ROM) on excursion distances while performing the Star Excursion Balance Test (SEBT). The SEBT measures dynamic postural control. Participants performed three trials of the SEBT in each of eight directions while balancing on the right and left legs, respectively. The SEBT found no statistically significant relations between foot type or ROM and the excursion distances. Significant correlations were revealed between height and excursion distance and leg length and excursion distance, with leg length having the stronger correlation. Using raw excursion measures, men were found to have substantially greater excursion distances than females. However, after normalizing 
excursion distances to leg length, there were no substantial differences related to sex. Thus, when using the SEBT for experimental or clinical purposes, participants' excursion distances should be normalized to leg length to allow a more accurate comparison of performance among participants.

Use of force plates is popular for addressing biomechanics, and a number of studies have used this measurement method for balance analysis [47-50]. Torry et al. [51] described an interesting approach to drop landing analysis. They used biplane fluoroscopy to measure 3D rotations and translations of healthy knees during stiff drop landings to determine the relations between 3D rotations and anterior and lateral tibial translations. Attainment of balance can be analyzed not only via ground reaction force or EMG measurements, but by observing eCOG repositioning, as shown by Colby et al. [52]. In addition to ground reaction force, they focused on developing a functional test to measure dynamic stability that could differentiate between the injured and uninjured lower limbs, They tested two populations-individuals with ACL deficiency and those who had undergone ACLR - to establish the reliability of the test.

With regard to the current state of the art, our study provides new information on horizontal components of ground reaction force and eCOG relocation. Balance during the landing phase is different for healthy people and patients after ACLR. Our patients had greater eCOG fluctuations. The ground reaction horizontal components of the force ratio were greater for the patients than for the controls.

\section{Conclusions}

The horizontal ground reaction forces and eCOG positioning are appropriate parameters for evaluating the performance of the human biomechanical system during jump-down. They can be used to recognize an ACL injury as well as to evaluate progress during rehabilitation after ACLR.

\section{References}

[1] Bjordal, J. M., et al.: Epidemiology of Anterior Cruciate Ligament Injuries in Soccer, The American Journal of Sports Medicine 25.3 (1997) pp. 341345

[2] Ireland, M. L.: Anterior Cruciate Ligament Injury in Female Athletes: Epidemiology, Journal of Athletic Training 34.2 (1999) p. 150

[3] Hootman, J. M., Dick, R., \& Agel, J.: Epidemiology of Collegiate Injuries for 15 Sports: Summary and Recommendations for Injury Prevention Initiatives, Journal of Athletic Training 42.2 (2007) p. 311

[4] Frigo, C., Shiavi, R.: Applications in Movement and Gait Analysis, In: Electromyography Physiology, Engineering, and Noninvasive Applications, (2004) pp. 381-397 
[5] Beard, D. J., Soundarapandian, R. S., O'Connor, J. J., \& Dodd, C. A. F.: Gait and Electromyographic Analysis of Anterior Cruciate Ligament Deficient Subjects. Gait \& Posture, 4.2 (1996), pp. 83-88

[6] Boulgouris, N. V., Chi, Z. X.: Gait Recognition Using Radon Transform and Linear Discriminant Analysis, IEEE Trans on Image Proc, 16.3 (2007) pp. 731-740

[7] Kale, A., Sundaresan, A., Rajagopalan, A. N., Cuntoor, N. P., RoyChowdhury, A. K., Krüger, V., \& Chellappa, R.: Identification of Humans using Gait, IEEE Trans on Image Proc, 13.9 (2004) pp. 1163-1173

[8] Begg, R. K., Palaniswami, M., \& Owen B.: Support Vector Machines for Automated Gait Classification, IEEE Trans on Biomedical Eng, 52.5 (2005) pp. 828-838

[9] Kamruzzaman, J., \& Begg, R. K.: Support Vector Machines and Other Pattern Recognition Approaches to the Diagnosis of Cerebral Palsy Gait, IEEE Trans on Biomedical Eng, 53.12 (2006) pp. 2479-2490

[10] Yam, C. Y., Nixon, M. S., Carter, J. N.: Extended Model-based Automatic Gait Recognition of Walking and Running, Proc. of $3^{\text {rd }}$ Int. Conf. on Audioand Video-based Biometric Person Authentication (2001) pp. 278-283

[11] Hansen, M., Haugland, M. K., \& Sinkjær, T.: Evaluating Robustness of Gait Event Detection Based on Machine Learning and Natural Sensors, IEEE Trans on Neural Systems and Rehabilitation Eng, 12.1 (2004) pp. 8188

[12] Corazza, S., Mündermann, L., Chaudhari, A. M., Demattio, T., Cobelli, C., \& Andriacchi, T. P.: A Markerless Motion Capture System to Study Musculoskeletal Biomechanics: Visual Hull and Simulated Annealing Approach. Annals of Biomedical Engineering, 34.6 (2006) pp. 1019-1029

[13] Drosou, A., Ioannidis, D., Moustakas, K., \& Tzovaras, D.: Spatiotemporal Analysis of Human Activities for Biometric Authentication, Computer Vision and Image Understanding, 116.3 (2012) pp. 411-421

[14] Knoll, Z., Kiss, R. M., \& Kocsis, L.: Gait Adaptation in ACL Deficient Patients before and after Anterior Cruciate Ligament Reconstruction Surgery. Journal of Electromyography and Kinesiology, 14.3 (2004) pp. 287

[15] Tashman, S., Anderst, W., Kolowich, P., Havstad, S., \& Arnoczky, S. Kinematics of the ACL-Deficient Canine Knee during Gait: Serial Changes over Two Years. Journal of Orthopaedic Research, 22.5 (2004) pp. 931-941

[16] Devita, P., et al.: Gait Adaptations before and after Anterior Cruciate Ligament Reconstruction Surgery, Medicine and Science in Sports and Exercise, 29.7 (1997) p. 853 
[17] Tashman, S., Kolowich, P., Collon, D., Anderson, K., \& Anderst, W.: Dynamic Function of the ACL-reconstructed Knee during Running. Clinical Orthopaedics and Related Research, 454 (2007) pp. 66-73

[18] Tashman, S., Collon, D., Anderson, K., Kolowich, P., \& Anderst, W.: Abnormal Rotational Knee Motion during Running after Anterior Cruciate Ligament Reconstruction, The American Journal of Sports Medicine, 32.4 (2004) pp. 975-983

[19] Waite, J. C., Beard, D. J., Dodd, C. A. F., Murray, D. W., \& Gill, H. S.: In Vivo Kinematics of the ACL-Deficient Limb during Running and Cutting, Knee Surgery, Sports Traumatology, Arthroscopy, 13.5 (2005) pp. 377-384

[20] Decker, M. J., Torry, M. R., Wyland, D. J., Sterett, W. I., \& Richard Steadman, J.: Gender Differences in Lower Extremity Kinematics, Kinetics and Energy Absorption during Landing. Clinical Biomechanics, 18.7 (2003) pp. 662-669

[21] Savalli, L., Hernandez-Sendin, M. I., Soille, J., \& Laboute, E: Interest of the Monopodal Jump as an Indirect Means of Assessing Muscle Recovery Distance of an ACL Reconstruction, Annals of Physical and Rehabilitation Medicine, 55.12012 , p.e76

[22] Graham-Smith, P., \& Lees, A.: A Three-Dimensional Kinematic Analysis of the Long Jump Take-Off. Journal of Sports Sciences, 23.9 (2005) pp. 891-903

[23] Pflum, M. A., Shelburne, K. B., Torry, M. R., Decker, M. J., \& Pandy, M. G.: Model Prediction of Anterior Cruciate Ligament Force during DropLandings. Medicine and Science in Sports and Exercise, 36.11 (2004) p. 1949

[24] Pain, M. T., \& Challis, J. H.: The Influence of Soft Tissue Movement on Ground Reaction Forces, Joint Torques and Joint Reaction Forces in Drop Landings. Journal of Biomechanics, 39.1 (2006) pp. 119-124

[25] Kram, R., Griffin, T. M., Donelan, J. M., \& Chang, Y. H.: Force Treadmill for Measuring Vertical and Horizontal Ground Reaction Forces. Journal of Applied Physiology, 85.2 (1998) pp. $764-769$

[26] Kutilek, Patrik, et al.: Kinematic Quantification of Gait Asymmetry Based on Characteristics of Angle-Angle Diagrams, Acta Polytechnica Hungarica 11.5 (2014)

[27] Bíró, I., \& Fekete, G.: Approximate Method for Determining the Axis of Finite Rotation of Human Knee Joint. Acta Polytechnica Hungarica, 11.9 (2014)

[28] Noyes, Frank R., et al.: The Drop-Jump Screening Test Difference in Lower Limb Control by Gender and Effect of Neuromuscular Training in 
Female Athletes, The American Journal of Sports Medicine 33.2 (2005) pp. 197-207

[29] Torry, Michael R., et al.: Knee Kinematic Profiles during Drop Landings: a Biplane Fluoroscopy Study, Medicine and Science in Sports and Exercise, 43.3 (2011) pp. 533-541

[30] Horita, T., et al.: Stretch Shortening Cycle Fatigue: Interactions among Joint Stiness, Reflex, and Muscle Mechanical Performance in the Drop Jump, European Journal of Applied Physiology and Occupational Physiology 73.5 (1996) pp. 393-403

[31] Russell, Kyla A., et al.: Sex Differences in Valgus Knee Angle during a Single-Leg Drop Jump, Journal of Athletic Training 41.2 (2006) p. 166

[32] Graham-Smith, P., \& Lees, A. A Three-Dimensional Kinematic Analysis of the Long Jump Take-Off. Journal of Sports Sciences, 23.9 (2005) pp. 891903

[33] Fagenbaum, R., \& Darling, W. G.: Jump Landing Strategies in Male and Female College Athletes and the Implications of Such Strategies for Anterior Cruciate Ligament Injury, The American Journal of Sports Medicine, 31.2 (2003) pp. 233-240

[34] Withrow, T. J., et al.: The Effect of an Impulsive Knee Valgus Moment on in Vitro Relative ACL Strain during a Simulated Jump Landing: Clinical Biomechanics 21.9 (2006) pp. 977-983

[35] Pappas, E., et al.: Biomechanical Differences between Unilateral and Bilateral Landings from a Jump: Gender Differences, Clinical Journal of Sport Medicine 17.4 (2007) pp. 263-268

[36] McKinley, P., \& Pedotti, A.: Motor Strategies in Landing from a Jump: The Role of Skill in Task Execution. Experimental Brain Research, 90.2 (1992) pp. 427-440

[37] Horstmann, G. A., F. Huethe, and V. Dietz.: Special Treadmill for the Investigation of Standing and Walking in Research and in Clinical Medicine."Biomedizinische Technik. Biomedical Engineering 32.10 (1987) 250-254

[38] Normand, M. C., Normand, A. R., \& Marchand, D.: Muscular Responses Comparison between Normal and Acl Reconstructed Knees during Drop Jump. Journal of Biomechanics, 25.7 (1992) p. 696

[39] Waite, J. C., et al.: In Vivo Kinematics of the ACL-Deficient Limb during Running and Cutting, Knee Surgery, Sports Traumatology, Arthroscopy 13.5 (2005) pp. 377-384

[40] Melińska, A. et al.: The Method of Evaluation of Biomechanical Parameters of Human Lower Limbs in the Jump from Steps of Different Heights, Fizjoterapia Polska, 11.4 (2011) pp. 327-340 
[41] Czamara, A.: Evaluation of Physiotherapeutic after Endoscopic Reconstruction of the Anterior Cruciate Ligament of the Knee, The Doctoral Dissertation. University of Physical Education in Warsaw, Warsaw, Poland, 2006, pp. 39-82

[42] Melinska, A. et al.: Biomechanical Characteristics of the Jump Down of Healthy Subjects and Patients with Lower Limbs Dysfunctions, Acta of Bioengineering and Biomechanics, article in press: DOI: 10.5277/ABB00208-2014-04

[43] Paterno, M. V., et al.: Limb Asymmetries in Landing and Jumping 2 Years Following Anterior Cruciate Ligament Reconstruction, Clinical Journal of Sport Medicine, 17.4 (2007) pp. 258-262

[44] Ortiz, A. et al.: Landing Mechanics between Noninjured Women and Women with Anterior Cruciate Ligament Reconstruction during 2 Jump Tasks, The American Journal of Sports Medicine, 36.1 (2008) pp. 149-157

[45] Decker, M. J. et al.: Landing Adaptations after ACL Reconstruction. Medicine and Science in Sports and Exercise, 34.9 (2002) p. 1408

[46] Gribble, P. A., \& Hertel, J.: Considerations for Normalizing Measures of the Star Excursion Balance Test. Measurement in Physical Education and Exercise Science, 7.2 (2003) pp. 89-100

[47] Waite, J. C., et al.: In Vivo Kinematics of the ACL-Deficient Limb during Running and Cutting, Knee Surgery, Sports Traumatology, Arthroscopy 13.5 (2005) pp. 377-384

[48] Davis, Roy B., et al.: A Gait Analysis Data Collection and Reduction Technique." Human Movement Science 10.5 (1991) pp. 575-587

[49] Pain, M. T., \& Challis, J. H.: The Influence of Soft Tissue Movement on Ground Reaction Forces, Joint Torques and Joint Reaction Forces in Drop Landings, Journal of Biomechanics, 39.1 (2006) pp. 119-124

[50] Kram, Rodger, et al.: Force Treadmill for Measuring Vertical and Horizontal Ground Reaction Forces, Journal of applied physiology 85.2 (1998) pp. 764-769

[51] Riley, P. O., et al.: A Kinematic and Kinetic Comparison of Overground and Treadmill Walking in Healthy Subjects, Gait \& posture 26.1 (2007) pp. $17-24$

[52] Colby, S. M., et al.: Lower Limb Stability with ACL Impairment, The Journal of Orthopaedic and Sports Physical Therapy 29.8 (1999) pp. 444-51 